\title{
Comment
}

\section{The role by scientific publications in science communication}

\author{
Martha Fabbri
}

In their contributions to this special issue, the British science writer Jon Turney and the American scholar Bruce Lewenstein discuss the validity of the book as a means for science communication in the era of the Internet, whereas the article by Vittorio Bo deals with scientific publishing in a broader sense.

As regards the issue of the books, Turney and Lewenstei have mirror-like points of view: the former dwells upon its construction, the second on its effect within different communities of readers. And yet, both writers need to quote, among others, two remarkable and identical cases: Cosmos, by the great populariser Carl Sagan (the Italian edition, Cosmo, is out of print, but if you are interested in it, you may still read it, just apropos, on the Internet) and From The Big Bang to Black Holes by Stephen Hawking (still published in the Bur pocket-edition). This is not a coincidence: it this is the manifestation of a common scientific culture that is derived also - and, as stressed by the three authors themselves in the case of non-specialists, especially - from one's reading experiences.

If popularising books, as suggested by Lewenstein and supported by Bo, do shape our conception of science, create scientific vocations and sometimes contribute to the intellectual development of science itself, then it is important to wonder whether and what authors - Italian or foreign ones - have had a similar influence on the scientific culture of our country.

The same applies to school textbooks too. Let's consider the Amaldi physics coursebook for high schools, published by Zanichelli and adapted from a coursebook for the 'Liceo' secondary school written by Enrico Fermi in 1929: it is obvious to stress that the evolution of this text over the years has been the result of an adjustment to new teaching models, but it may be useful to try to think whether and how, on the contrary, it was this widespread coursebook that has had an influence on the conception of physics in Italy.

Turney says that «the Anglo-American boom in popular science publishing has certainly faded». Let's mention a few examples, restricted to books. In the past five years only, Italy saw the birth of different publishing initiatives totally devoted - or very "oriented" - to science: Bruno Mondadori with the series entitled Matematica e Dintorni in 2002, Codice, Orme and Sironi in 2003, the SciBooks publishing house in 2005, the Dimensioni della Scienza series published by Bompiani in 2006.

Certainly, a very wide range of novelties does not necessarily imply large numbers in terms of sales. Yet 2004 saw the explosion of the Odifreddi "case", whose Le Menzogne di Ulisse saw a ten-fold increase in sales compared to the "usual" Italian essay's figures.

On the other hand, it is now commonly agreed that From The Big Bang to Black Holes was bought by everybody, but honestly read by nobody (as confirmed also by Lewenstein). So, how should one know whether the figures in sales recorded by Odifreddi, Margherita Hack or Piero Angela really make a difference? Nothing is left but asking the readers.

Translated by Massimo Caregnato

\begin{abstract}
Author
Martha Fabbri has a degree in Physics and is the editor to the scientific popularising series 'Galápagos' for Sironi Editore, a member of the Scientific Committee and a lecturer for the Master in Science Communication at SISSA-ISAS. E-mail:martha_fabbri@alphatest.it
\end{abstract}

